\title{
Level of fibrinogen and risk of fatal and non-fatal stroke. EUROSTROKE: a collaborative study among research centres in Europe
}

\author{
M L Bots, P C Elwood, J T Salonen, A Freire de Concalves, J Sivenius, A Di Carlo, \\ Y Nikitin, V Benetou, J Tuomilehto, P J Koudstaal, D E Grobbee
}

J Epidemiol Community Health 2002;56(Suppl I):i14-i18

See end of article for authors' affiliations

Correspondence to Dr M L Bots, Julius Centre for General Practice and Patient Oriented Research, University Medical Centre Utrecht, room D01.335,

Heidelberglaan 100, 3584 CX Utrecht, the

Netherlands;

M.L.Bots@jc.azu.nl

Accepted for publication 30 July 2001 t is well established that raised levels of fibrinogen increase the risk of coronary heart disease. This has convincingly been shown in population-based studies among apparently healthy subjects. ${ }^{1-6}$ Also, among subjects with a history of coronary heart disease fibrinogen is an important predictor of future coronary events. ${ }^{7}$ Studies on the association between fibrinogen and stroke are, however, limited. ${ }^{138}$ These studies were based on a limited number of events and in general restricted to overall stroke without subtyping the stroke events.

We evaluated the association between fibrinogen and fatal, non-fatal, haemorrhagic and ischaemic stroke combining data obtained in three European cohorts participating in EUROSTROKE.

\section{METHODS}

The rationale and design of EUROSTROKE have been described in detail elsewhere. ${ }^{9}$ In short, EUROSTROKE is a collaborative study among European research centres to investigate (1) the variation in incidence of fatal and non-fatal ischaemic and haemorrhagic stroke among populations in different European countries; (2) whether the observed differences in stroke incidence across countries can be explained by differences in prevalence of established cardiovascular risk factors; (3) the relative importance of smoking and some selected dietary factors (potassium intake, alcohol consumption), haemostatic disturbances (fibrinogen) and comorbidity (rheumatic heart disease, atrial fibrillation) compared with established risk factors as determinants of the occurrence of ischaemic and haemorrhagic stroke. The
EUROSTROKE database is drawn from ongoing European population-based prospective follow up studies (cohorts) and is designed as a case-control study nested within these ongoing studies. For each stroke case, two controls were sampled. Controls were matched on day of baseline examination only. Apart from its objectives, the EUROSTROKE database allows for aetiological analyses looking into various risk factors for stroke. EUROSTROKE formally started on 1 January 1994. At present, fibrinogen data from three cohorts were available for analysis.

\section{Finland}

The Finnish contribution to EUROSTROKE comes from the Kuopio Ischemic Heart Disease Risk Factor study, which is a population-based prospective cohort study comprised of an age stratified random sample of 2682 men aged 42, 48, 54, and 60 years. The baseline examination was performed between 1984 and $1989 .{ }^{10}$ Fatal and non-fatal stroke cases were collected through the national mortality statistics and the FINMONICA stroke registries. Stroke was defined according to FINMONICA criteria and definitions. ${ }^{11}$ Case ascertainment from the baseline examination to I January 1993 revealed 74 stroke cases. Controls subjects $(n=148)$ were randomly drawn from the cohort that remained free from stroke during follow up. Fibrinogen was measured in 61 cases and 124 controls.

\section{The Netherlands}

The Dutch contribution to EUROSTROKE comes from the Rotterdam Study, which is a population-based prospective follow up study among 7983 subjects, aged 55 years or over, living in the suburb of Ommoord in Rotterdam, the 
Netherlands. ${ }^{12}$ Baseline data were collected from March 1990 to July 1993. In the Rotterdam Study, information on incident fatal and non-fatal events is obtained from the general practitioners (GPs) working in the study district of Ommoord as described earlier. ${ }^{13}$ The GPs involved report all possible cases of stroke to the Rotterdam research centre. Events are presented in coded information following the International Classification of Primary Care (ICPC) ${ }^{14}$ With respect to the vital status of the participants, information is obtained at regular intervals from the municipal authorities in Rotterdam and also death of a participant is reported by GPs. When an event or death has been reported, additional information is obtained by interviewing the GP and scrutinising information from hospital discharge records in case of admittance or referral. All suspected cerebrovascular events reported by the GPs were submitted for review to the EUROSTROKE case review board. From baseline to December 1994, 192 stroke cases were identified and submitted for review and a total of 384 control subjects were drawn from the remainder of the cohort that remained free from stroke during follow up. Altogether 157 events were classified as definite or probable strokes. As fibrinogen was measured in the first 2511 participants only in the Rotterdam Study, ${ }^{15}$ fibrinogen measurements were available in 62 cases and 203 control subjects.

\section{United Kingdom}

The British contribution to EUROSTROKE comes from the Caerphilly Heart Disease study in Wales, United Kingdom, in which 2512 men, aged 45 to 59 years are participating. ${ }^{16}$ Baseline examinations took place from 1979 to 1983. Follow up examinations were performed from 1984 to 1988 (phase II) and from 1989 to 1993 (phase III). Stroke events were registered through national mortality statistics, hospital discharge records, self report, and family report. Of the registered events, additional information on signs and symptoms, on neuroimaging, necropsy and a copy of the discharge records were collected. When complete, stroke cases were submitted for review to the EUROSTROKE case review board as descried earlier. Recently, 100 stroke events had been submitted for review and 200 controls subjects had been drawn from the remaining cohort. Eighty four stroke cases were classified as definite/probable stroke by the EUROSTROKE review board. Data on fibrinogen were present for 79 cases and 194 controls.

\section{EUROSTROKE case review board}

The review board comprised four Dutch neurologists. Based on all information, including symptoms and signs obtained by interviewing the GP or, in case of hospital referral, hospital data, the neurologists classified the events as definite, probable and possible stroke. Events were classified by two neurologists. In case of disagreement a third neurologist was consulted, whose opinion was decisive for the final classification. This analysis is restricted to definite and probable events. For the present analysis an incident stroke was considered to have occurred when (1) the event had led to a hospitalisation and the hospital discharge record indicated a diagnosis of a new stroke. The clinical diagnosis was based on signs and symptoms, and neuroimaging investigations during hospital stay (definite stroke); or (2) in case of no hospitalisation, signs and symptoms associated with the event obtained from the GP records and interview were highly suggestive of a stroke according to the neurologists (probable stroke) or (3) in case of out hospital death, when the GPs reported that the cause of death was a cerebrovascular accident and a cardiac cause was judged by the GP to be highly unlikely (probable stroke).

The event was classified into first and recurrent stroke and into subarachnoid haemorrhage, intracranial haemorrhage, intracerebral infarction, and unspecified (not documented).

\section{Key points}

- Increased fibrinogen increases the risk of coronary heart disease.

- Increased fibrinogen is a strong predictor of stroke.

- The association holds for fatal and non-fatal stroke, ischaemic and haemorrhagic stroke.

For the non-MONICA centres (the Netherlands, United Kingdom) ischaemic stroke was further classified into lacunar infarct; total anterior circulation infarct; partial anterior circulation infarct; posterior circulation infarct, and unspecified infarct (undocumented). The classification was made following the guidelines described by Bamford and coworkers ${ }^{17}$ and by the European Atrial Fibrillation Trial. ${ }^{18}$

\section{Cardiovascular risk factors}

As EUROSTROKE is based on ongoing cohort studies, information on cardiovascular risk factors in each of the participating centres was already being collected before the EUROSTROKE project was established. Whenever possible, an exhaustive attempt was made to further harmonise the collected information to make comparison across studies possible. Nevertheless, baseline measurements could not be standardised beyond the attempts done in each individual study.

In each of the centres information on smoking, alcohol consumption, and medical history was obtained by questionnaire. The subject's smoking behaviour was categorised into current, former or never. Alcohol consumption was categorised into current drinkers and non-current drinkers (former and never). In addition, an estimate of grams of alcohol per day was obtained. Presence of diabetes mellitus was generally based on the question "Do you suffer from diabetes mellitus?". In the Rotterdam study diabetes mellitus was considered present when subjects used blood sugar lowering drugs. Information on a history of stroke was obtained by direct questioning at baseline "Did you ever suffer from a stroke?". A similar approach was taken for myocardial infarction. Presence of angina pectoris was based on either the cardiovascular Rose questionnaire or direct questioning.

In general, systolic and diastolic blood pressure were measured twice at one occasion in the sitting position. In Cardiff, only one blood pressure measurement was performed. Height and weight were measured and body mass index (kg/ $\mathrm{m}^{2}$ ) was calculated. In all four centres an electrocardiogram was made and the presence of a Q-wave myocardial infarction and left ventricular hypertrophy were assessed according to the Minnesota classification system, or in Rotterdam, by using the automated diagnostic classification system of the Modular Electrocardiogram Analysis System (MEANS). ${ }^{19} 20$ Apart from Rotterdam, a fasting blood sample was taken for determination of serum lipids (total cholesterol, HDL cholesterol). Serum total cholesterol level was determined with an automatic analyser using enzymatic procedures in Rotterdam, ${ }^{21}$ in Cardiff, ${ }^{22}$ in Finland, ${ }^{23}$ and Novosibirsk. ${ }^{24}$ Fibrinogen was measured according to the Von Clauss method ${ }^{25}$ in Kuopio, according to the nephelometric method in Cardiff and Rotterdam fibrinogen levels were derived from the clotting curve of the prothrombin time assay using Thromborel S as reagent on an Automated Coagulation Laboratory. ${ }^{15}$ The latter method correlates well with the Von Clauss method. ${ }^{26}$

\section{Data analysis}

Complete data on both stroke and fibrinogen were available for Cardiff (79 cases/194 controls), Kuopio (61 cases/124 controls), and Rotterdam (62 cases/203 controls). As potential confounders systolic blood pressure, diastolic blood pressure, 
Table 1 General characteristics of the study population

\begin{tabular}{|c|c|c|c|c|}
\hline Characteristic & Cardiff, UK & Kuopio, FIN & Rotterdam, NL & All \\
\hline Case/control & $79 / 194$ & $61 / 124$ & $62 / 203$ & $202 / 521$ \\
\hline Age (y) & $57.5(5.9)$ & $55.2(4.4)$ & $73.4(9.0)$ & $62.7(10.7)$ \\
\hline Female $(\%)$ & * & * & 64.5 & 23.6 \\
\hline Systolic pressure (mm Hg) & $146(22)$ & $132(18)$ & $143(23)$ & $141(22)$ \\
\hline Diastolic pressure $(\mathrm{mm} \mathrm{Hg})$ & $86.4(12.8)$ & $88.3(10.0)$ & $72.2(12.1)$ & $81.9(13.8)$ \\
\hline Total cholesterol (mmol/l) & $5.7(1.2)$ & $6.1(1.2)$ & $6.6(1.2)$ & $6.2(1.3)$ \\
\hline HDL cholesterol (mmol/l) & $1.05(0.33)$ & $1.26(0.28)$ & $1.36(0.37)$ & $1.26(0.37)$ \\
\hline Current smoking $(\%)$ & 46.1 & 25.4 & 24.5 & 33.0 \\
\hline Body mass index $\left(\mathrm{kg} / \mathrm{m}^{2}\right)$ & $26.6(3.7)$ & $26.9(3.4)$ & $26.4(3.8)$ & $26.6(3.6)$ \\
\hline Diabetes mellitus (\%) & 3.6 & 6.4 & 9.1 & 6.4 \\
\hline Fibrinogen (g/l) & $4.04(0.95)$ & $3.20(0.62)$ & $2.88(0.71)$ & $3.40(0.94)$ \\
\hline
\end{tabular}

Values are unadjusted proportions or means with standard deviations in parentheses. ${ }^{*}$ Only men participated in the study.

Table 2 Stroke characteristics of the participating centres

\begin{tabular}{lllll}
\hline Characteristic & $\begin{array}{l}\text { Cardiff, } \\
\text { UK }\end{array}$ & $\begin{array}{l}\text { Kuopio, } \\
\text { FIN }\end{array}$ & $\begin{array}{l}\text { Rotterdam, } \\
\text { NL }\end{array}$ & All \\
\hline Total stroke & 79 & 91 & 62 & 202 \\
Fatal & 23 & 10 & 15 & $48(23.8 \%)$ \\
Non-fatal & 56 & 51 & 47 & $154(76.2 \%)$ \\
Haemorrhagic & 11 & 11 & 3 & $25(12.4 \%)$ \\
Ischaemic & 53 & 45 & 40 & $138(68.3 \%)$ \\
Unspecified & 15 & 5 & 19 & $39(19.3 \%)$ \\
\hline
\end{tabular}

Values are absolute numbers.

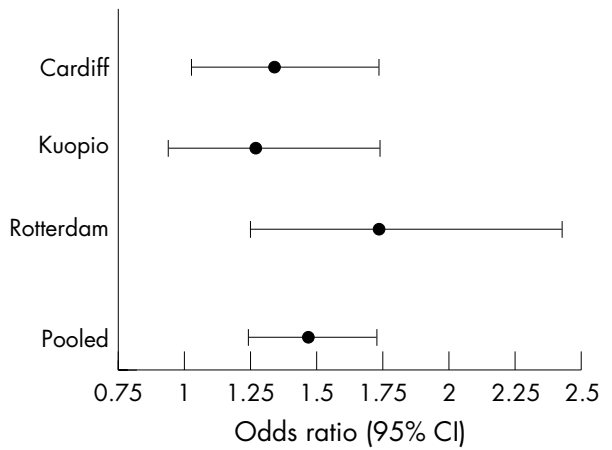

Figure 1 Risk of stroke per quartile increase in fibrinogen, adjusted for age, sex, smoking, and systolic blood pressure.

hypertension, serum total and HDL cholesterol, body mass index, current smoking and diabetes mellitus were evaluated using logistic regression analysis. Logistic analysis was applied to study the association between fibrinogen and stroke. Separate analyses were performed for total stroke, first ever stroke, fatal stroke (died within 28 days after onset), non-fatal stroke, haemorrhagic stroke, cerebral infarction and for cortical and lacunar stroke. Results are presented as odds ratios with corresponding 95\% confidence intervals (95\% CI).
The analyses were first performed for each centre separately with fibrinogen by $1 \mathrm{~g} / \mathrm{l}$ increase (continuous variable) and by quartiles (categorised variable, three dummy variables). Fibrinogen cut off points for Cardiff were 3.4, 4.0, and $4.6 \mathrm{~g} / \mathrm{l}$; for Kuopio 2.7, 3.2, and $3.5 \mathrm{~g} / \mathrm{l}$; and for Rotterdam 2.4, 2.8, 3.3 $\mathrm{g} / \mathrm{l}$. The same approach was taken for the analyses in which information from all four centres was combined (pooled analyses). Each individual was categorised into a quartile based on the centre specific fibrinogen distribution. Multiplicative interaction terms (fibrinogen $\times$ centre) and (fibrinogen $\times$ age) indicated no difference in associations between centres $(\mathrm{p}=0.98)$ and age $(\mathrm{p}=0.44)$.

\section{RESULTS}

General characteristics of the study populations are given in table 1. Characteristics of the stroke events are presented in table 2 . Among the fatal strokes, $18.8 \%$ were a haemorrhagic, $41.7 \%$ cerebral infarctions and $39.6 \%$ could not be specified with the available information. The proportions for non-fatal stroke were $10.4 \%, 76.6 \%$, and $13.0 \%$, respectively. In analyses in which information of all centres was combined systolic blood pressure, diastolic blood pressure, current smoking, and history of diabetes mellitus were positively, and significantly $(p<0.05)$ related to stroke. No associations were found for total and HDL cholesterol and body mass index. Significant direct associations with fibrinogen were found for age, systolic blood pressure, smoking, and inverse for HDL cholesterol. Diastolic pressure, body mass index and history of diabetes were not associated with fibrinogen. Therefore, systolic blood pressure and current smoking were considered as potential confounding variables and adjusted for in the analyses apart for age and sex.

When age, sex, smoking and systolic blood pressure were accounted for in the analyses the odds ratio for stroke per $1 \mathrm{~g} / \mathrm{l}$ increase in fibrinogen in Cardiff was 1.33 (95\% CI 1.00 to 1.79), in Kuopio 1.45 (95\% CI 0.85 to 2.45 ) and in Rotterdam 2.01 ( $95 \%$ CI 1.24 to 3.27). The odds ratio for stroke per centre specific fibinogen quartile is presented in figure 1 .

Table 3 describes the association between fibrinogen and risk of stroke for all centres combined. A graded increased risk

Table 3 Fibrinogen and risk of stroke*

\begin{tabular}{lllll}
\hline & Quartile II & Quartile III & Quartile IV & Trendt \\
\hline All strokes & $1.08(0.63$ to 1.84$)$ & $1.91(1.12$ to 3.26$)$ & $2.78(1.64$ to 4.72$)$ & $1.47(1.24$ to 1.73$)$ \\
First ever & $1.09(0.62$ to 1.91$)$ & $1.91(1.10$ to 3.34$)$ & $2.52(1.45$ to 4.41$)$ & $1.41(1.19$ to 1.68$)$ \\
Fatal & $0.57(0.21$ to 1.55$)$ & $1.16(0.45$ to 2.99$)$ & $2.30(0.96$ to 5.57$)$ & $1.47(1.08$ to 1.98$)$ \\
Non-fatal & $1.30(0.71$ to 2.9$)$ & $2.29(1.25$ to 4.19$)$ & $2.98(1.64$ to 5.43$)$ & $1.48(1.23$ to 1.77$)$ \\
Haemorrhagic & $2.17(0.41$ to 11.5$)$ & $6.76(0.94$ to 32.4$)$ & $6.59(1.31$ to 33.3$)$ & $1.85(1.22$ to 2.81$)$ \\
Ischaemic & $0.91(0.50$ to 1.65$)$ & $1.44(0.79$ to 2.62$)$ & $2.25(1.26$ to 4.02$)$ & $1.37(1.14$ to 1.65$)$ \\
Ischaemic & $0.99(0.57$ to 1.73$)$ & $1.64(0.94$ to 2.88$)$ & $2.54(1.47$ to 4.39$)$ & $1.42(1.20$ to 1.69$)$ \\
\hline
\end{tabular}

*Odds ratios relative to subjects with a fibrinogen in the first quartile of the centre specific distribution with $95 \%$ confidence intervals; $†$ odds ratio per quartile increase based on the centre specific distribution, adjusted for age, sex, smoking, systolic blood pressure; fincluding unspecified strokes. 
Table 4 Association of fibrinogen with stroke in strata of cardiovascular risk factors

\begin{tabular}{|c|c|c|c|}
\hline Characteristic & Subjects & Odds ratio & $95 \% \mathrm{Cl}$ \\
\hline \multicolumn{4}{|l|}{ Smoking } \\
\hline Yes & 233 & 1.36 & 1.05 to 1.77 \\
\hline No & 476 & 1.54 & 1.25 to 1.91 \\
\hline \multicolumn{4}{|l|}{ Diabetes mellitus } \\
\hline Yes & 46 & 1.90 & 0.98 to 3.67 \\
\hline No & 661 & 1.46 & 1.22 to 1.73 \\
\hline \multicolumn{4}{|l|}{ Myocardial infarction } \\
\hline Yes & 52 & 1.51 & 0.79 to 2.86 \\
\hline No & 647 & 1.44 & 1.21 to 1.72 \\
\hline \multicolumn{4}{|c|}{ HDL cholesterol (median $1.2 \mathrm{mmol} / \mathrm{l})$} \\
\hline$<$ median & 295 & 1.54 & 1.20 to 1.98 \\
\hline$\geqslant$ median & 414 & 1.42 & 1.13 to 1.76 \\
\hline \multicolumn{4}{|l|}{ Systolic pressure } \\
\hline$<120 \mathrm{~mm} \mathrm{Hg}$ & 98 & 1.21 & 0.73 to 2.00 \\
\hline $120-139 \mathrm{~mm} \mathrm{Hg}$ & 250 & 1.40 & 1.05 to 1.87 \\
\hline $140-159 \mathrm{~mm} \mathrm{Hg}$ & 220 & 1.41 & 1.05 to 1.88 \\
\hline$\geqslant 160 \mathrm{~mm} \mathrm{Hg}$ & 289 & 1.99 & 1.37 to 2.89 \\
\hline
\end{tabular}

of stroke is observed with increasing fibrinogen levels. The association, adjusted for other factors was similar for first and all strokes, for fatal and non-fatal ones, and for different subtypes. The odds ratio for lacunar infarction $(n=19)$ with an increase of fibrinogen per quartile was 1.36 (95\% CI 0.85 to $2.19)$, whereas the odds ratio for anterior cerebral infarction (total anterior circulation infarct + partial anterior circulation infarct, $\mathrm{n}=42$ ) was 1.25 (95\% CI 1.05 to 2.00 ).

The association between fibrinogen and stroke for different strata of cardiovascular risk factors is presented in table 4 . The associations do not seem to differ across strata of smoking, diabetes mellitus, previous myocardial infarction and HDL cholesterol. Stronger associations were observed with increasing systolic blood pressure, although not statistically significant $(\mathrm{p}>0.50)$.

\section{DISCUSSION}

This analysis of the EUROSTROKE project indicates that increased fibrinogen is a powerful predictor of stroke. Results did not disclose a differential relation with fatal or non-fatal stroke, or with type of stroke (ischaemic or haemorrhagic).

Several aspects of this study should be considered. Firstly, we cannot be sure about the completeness of case ascertainment in each study. The proportion fatal cases across the centres in this analyses differed from $11 \%$ in Kuopio to $29 \%$ in Cardiff (table 2). The latter may be indicative for underrepresentation of non-fatal cases. If the selection of non-fatal cases is associated with a lower or higher prevalence of the exposure of interest (fibrinogen), it may bias a true positive association towards a reduce or increased odds ratio or a null finding. As the risk related to fibrinogen was similar in fatal and non-fatal strokes, it is unlikely that the differences in the degree of case ascertainment would have caused a bias in the results. Strengths of this study are the fairly large number of well diagnosed and classified events for both men and women, presence of data on a large number of potential confounders, a wide age range, and risk factors assessment before the occurrence of the stroke event.

The only few population-based studies in which the association between fibrinogen and the risk of stroke was evaluated, ${ }^{13}$ were based on a relatively small number of events (37 and 92) and restricted to overall stroke only. Our observed odds ratios were similar to those reported by Wilhelmsen and coworkers $^{1}$ and Kannel and coworkers. ${ }^{3}$ The present analyses among 202 stroke events extends these findings by showing that a raised level of fibrinogen is associated with an increased risk of fatal and non-fatal stroke, and with ischaemic and haemorrhagic stroke. Furthermore, an explanatory analysis did not show major differences in the strength of the association with fibrinogen for lacunar and cortical cerebral infarctions. Findings were independent for established cardiovascular risk factors, in particular smoking and systolic blood pressure.

Several mechanisms have been described through which fibrinogen may increase the risk of development of atherosclerosis and of cardiovascular disease ${ }^{27}$ Central in most studies is whether increased fibrinogen is a cause of atherosclerotic disease or a consequence. Recently, van der Bom and coworkers convincingly showed that the "consequence" concept may be more applicable than the "cause" concept. ${ }^{28}$ Our finding that the magnitude association between fibrinogen and stroke tended to be stronger among those with high systolic blood pressure compared with low systolic blood pressure is supportive of that concept. A high fibrinogen level has been associated with coronary and carotid atherosclerosis. ${ }^{29}{ }^{30}$ Also a high systolic blood pressure may in part be attributed to increased arterial stiffness of the large arteries because of atherosclerotic changes. Hence, it is probable that among these patients are at considerable increased of stroke compared those with low pressure and lower fibrinogen levels. Furthermore, it may explain in part the positive association between fibrinogen and systolic blood pressure, as reported by others. ${ }^{31}$

In conclusion, this analysis of the EUROSTROKE project indicates that fibrinogen is a powerful predictor of stroke. Results did not disclose a differential relation with fatal or non-fatal stroke, or with type of stroke (ischaemic or haemorrhagic).

\section{ACKNOWLEDGEMENTS}

Funding: EUROSTROKE is supported by grant BMHl-CT93-1786 from the European Community BIOMED I programme and by grant CIPD-CT94-0256 from the European Community PECO programme.

We acknowledge four Dutch neurologists for their contribution to the classification of the stroke events: Jelis Boiten, University Hospital Maastricht; Jaap Kappelle, University Hospital Utrecht; Martien Limburg, Academic Medical Centre, Amsterdam; Peter J Koudstaal, University Hospital Rotterdam. We acknowledge Dr O Krüger of the Trondelhag Study in Norway for his participation in the EUROSTROKE project.

\section{Authors' affiliations}

M L Bots, D E Grobbee, Epidemiology and Biostatistics, Erasmus University Medical School, Rotterdam, the Netherlands

M L Bots, D E Grobbee, Julius Centre for General Practice and Patient

Oriented Research, University Medical Centre Utrecht, Utrecht, the Netherlands

P C Elwood, MRC Epidemiology Unit, Llandough Hospital, Penarth, South Glamorgan, UK

J T Salonen, Research Institute of Public Health, University of Kuopio, Kuopio, Finland

A Freire de Concalves, Neurology, Hospitais da Universidade de Coimbra, Coimbra, Portugal

J Sivenius, Department of Neurology, University of Kuopio, Kuopio, Finland

A Di Carlo, National Research Council of Italy (CNR-CSFET) Italian Longitudinal Study on Ageing, Florence, Italy

Y Nikitin, Russian Academy of Medical Sciences Siberian Branch, Institute of Internal Medicine, Novosibirsk, Russia

V Benetou, Hygiene and Epidemiology, University of Athens Medical School, Athens, Greece

J Tuomilehto, Epidemiology and Health Promotion, National Public Health Institute, Helsinki, Finland

P J Koudstaal, Neurology, University Hospital Rotterdam Dijkzigt, Rotterdam, the Netherlands

\section{REFERENCES}

1 Wilhelmsen L, Svardsudd K, Korsan-Bengtsen K, et al. Fibrinogen as a risk factor for stroke and myocardial infarction. N Engl J Med 1984;311:501-5. 
2 Stone MC, Thorp JM. Plasma fibrinogen - a major coronary risk factor. J $R$ Coll Gen Prac 1985;35:565-9.

3 Kannel WB, Wolf PA, Castelli WP, et al. Fibrinogen and risk of cardiovascular disease. The Framingham Study. JAMA

1987:258:1 183-6.

4 Meade TW, Mellows S, Brozvic M, et al. Haemostatic function and ischaemic heart disease: principal results of the Northwick Park Heart study. Lancet 1986;ii:533-7.

5 Yarnell JWG, Baker IA, Sweetnam PM, et al. Fibrinogen, viscosity, and white blood cell count are major risk factors for ischemic heart disease. The Caerphilly and Speedwell Collaborative Heart Disease studies. Circulation 1991;83:836-44.

6 Heinrich J, Balleisen L, Schulte H, et al. Fibrinogen and factor VII in the prediction of coronary risk. Results from the PROCAM study in healthy men. Arterioscler Thromb 1994;14:54-9.

7 Thompson SG, Kienast J, Pyke SDM, et al, for the European Concerted Action on Thrombosis and Disabilities Angina Pectoris study group. Hemostatic factors and the risk of myocardial infarction or sudden death in patients with angina pectoris. N Engl J Med 1995;332:635-41.

8 Bots ML, Hoes AW, Koudstaal PJ, et al. Fibrinogen predicts stroke. The Rotterdam Study. [Abstract]. Circulation 1996;88:1257.

9 Grobbee DE, Koudstaal PJ, Bots ML, et al. Incidence and risk factors for ischaemic and haemorrhagic stroke in Europe. EUROSTROKE: A collaborative study among research centers in Europe: rationale and design. Neuroepidemiology 1996;15:291-300.

10 Salonen JT, Salonen R, Seppänen K, et al. High density lipoprotein, $\mathrm{HDL}_{2}$ and $\mathrm{HDL}_{3}$ subfractions and the risk of acute myocardial infarction: a prospective population study in Eastern Finnish men. Circulation 1992;84:129-39.

11 Tuomilehto J, Sarti C, Narva EV, et al. The FinMonica stroke register. Community-based stroke registration and analysis of stroke incidence in Finland, 1983-1985. Am J Epidemiol 1992;135:1259-70.

12 Hofman A, Grobbee DE, Jong PTVM de, et al. Determinants of disease and disability in the elderly. The Rotterdam Elderly Study. Eur J Epidemiol $1991 ; 7: 403-22$

13 Bots ML, Hoes AW, Koudstaal PJ, et al. Common carotid intima-media thickness and risk of stroke and myocardial infarction. The Rotterdam Study. Circulation 1997;96:1432-7

14 Lamberts H, Wood M, Hofmans-Okkes I. The international classification of primary care in the European Community. Oxford: Oxford University Press, 1991

15 Bom JG van der, Maat MPM de, Bots ML, et al. Seasonal variation in fibrinogen. The Rotterdam Study. Thromb Haemost 1997;78:1059-62.
16 Caerphilly and Speedwell collaborative heart disease studies. The Caerphilly and Speedwell Collaborative Group. J Epidemiol Community Health 1984;38:259-62.

17 Bamford J, Sandercock P, Dennis M, et al. Classification and natural history of clinically identifiable subtypes of cerebral infarction. Lancet 1991;i:1521-6

18 European Atrial Fibrillation Trial Study Group. Secondary prevention in non-rheumatic atrial fibrillation after transient ischaemic attack or minor stroke. Lancet 1993;342: 1255-62.

19 Van Bemmel JH, Kors JA, van Herpen G. Methodology for the Modular Electrocardiogram Analysis System (MEANS). Methods Inf Med 1990;29:346-53.

20 Willems JL, Abreu-Lima C, Arnaud P, et al. The diagnostic performance of computer programs for the interpretation of electrocardiograms. $N$ Engl J Med 1991;325:1767-73.

21 VanGent CM, VanderVoort HA, de Bruijn AM, et al. Cholesterol detrminations. A comparative study of methods with special reference to enzymatic procedures. Clin Chem Acta 1977;75:243-51.

22 Steele BW, Koehler DF, Kuba K, et al. An enzymatic approach to ipoprotein quatification. Am J Clin Pathol 1980;73:75-8.

23 Nissinen A, Kivelä SL, Pekkanen J, et al. Levels of some biological risk indicators among elderly men in Finland. Age Ageing 1985;15:203-11.

24 US National Institute of Health. Manual of laboratory operations of the lipid research clinics program. Lipids and lipoprotein analysis. Washington DC: Government Printing Office Publication, no NIH, 1974:75-628.

25 Clauss A. Gerinnungsphysiologische schnellmethode zur bestimmung des fibrinogens. Acta Haematol 1957:17:237-46.

26 Rossi E, Mondonico P, Lombardi A, et al. Methods for determination of functional (clottable) fibrinogen by the new ACL coagulometers. Thromb Res 1988;52:453-68

27 Ernst E. Plasma fibrinogen- an independent cardiovascular risk factor. $J$ Intern Med 1990;227:365-72.

28 Bom JG van der, Maat MPM de, Bots ML, et al. Elevated fibrinogen: cause of consequence. Arterioscl Thromb Vasc Biol 1998:18:621-5.

29 ECAT Angina Pectoris Study Group. Baseline associations of haemostatic factors with extent of coronary arteriosclerosis and other coronary risk factors in 3000 patients with angina pectoris undergoing coronary angiography. Eur Heart J 1993;14:8-17.

30 Bots ML, Breslau PJ, Briët $E$, et al. Cardiovascular determinants of carotid artery disease: The Rotterdam Elderly Study. Hypertension 1992;19:717-20.

31 Folsom AR, Qamhieh HT, Hilner JE, et al, for the investigators of the Coronary Artery Risk Development in Young Adults (CARDIA) study. Am J Epidemiol 1993;138:1023-36. 